\title{
Genetics of high-dose methotrexate-induced oral mucositis: current perspectives
}

\author{
Sandra G Heil*,1 \\ ${ }^{1}$ Department of Clinical Chemistry, Erasmus MC University Medical Center Rotterdam, PO Box 2040, 3000 CA Rotterdam, The \\ Netherlands \\ *Author for correspondence: s.heil@erasmusmc.nl \\ "Ga large number of patients suffer from toxicity such as oral mucositis, which occurs in about $20 \%$ \\ of the patients treated with HD-MTX"
}

First draft submitted: 23 April 2019; Accepted for publication: 3 May 2019; Published online: 28 June 2019

Keywords: methotrexate $\bullet$ mucositis $\bullet$ pharmacogenetics $\bullet$ toxicity

High-dose methotrexate (HD-MTX) is part of routine care to patients with childhood and adult cancers such as lymphoma and acute-lymphoblastic leukemia. MTX is a folate-antagonist and inhibits the folate pathway leading to impaired DNA synthesis and cell death. Overall survival rate has increased dramatically since the introduction of HD-MTX to standard care. However, a large number of patients suffer from toxicity such as oral mucositis, which occurs in about $20 \%$ of the patients treated with HD-MTX [1]. Folinic acid (a folate vitamer) is prescribed to reduce toxic side effects of HD-MTX therapy. Identification of genetic variants that can predict occurrence of mucositis would aid in lowering toxicity and increased survival of patients treated with HD-MTX.

\section{Methotrexate uptake \& metabolism}

MTX is taken up by the reduced folate carrier (SLC19A1) and PCFT and is polyglutamated intracellularly by FPGS leading to up to seven polyglutamated chains (MTX-PG1-7) [2]. This polyglutamation of MTX renders MTX in the cell, which improves efficacy but also increases toxicity, as higher intracellular MTX levels can be reached [2]. MTX is most efficiently exported as MTX short chain polyglutamates (MTX PG1-3), and therefore, long-chain polyglutamates (MTX PG4-7) first need to be cleaved by GGH before they can be exported by ABC-transporters [2]. Intracellularly, MTX inhibits DHFR and TYMS; thereby, impairing purine- and pyrimidine synthesis, which leads to apoptosis of tumor cells. Folinic acid is a folate derivative which bypasses the DHFR block by MTX, and thereby, decreases toxicity rates of healthy cells. As MTX inhibits DHFR, this also has implications for one-carbon metabolism as less one-carbon groups are available for remethylation of homocysteine into methionine, and subsequently, availability of methyl groups for cellular methylation reactions [3].

\section{Pharmacogenetics of high-dose methotrexate in relation to oral mucositis}

Most candidate studies have focused at genes involved in pharmacokinetics (PK) and pharmacodynamics (PD) of HD-MTX in relation to mucositis. One of the most studied variants is the c.677 C>T variant in the gene coding for MTHFR (rs1801133). MTHFR 677 TT genotype has been associated with lower circulating folate levels, and therefore, is a good candidate gene to study in regard to MTX toxicity [4]. Several studies investigated the MTHFR c. $677 \mathrm{C}>\mathrm{T}$ variant in relation to oral mucositis but results are inconsistent. A recent meta-analysis demonstrated a significant association of the MTHFR $677 \mathrm{~T}$ allele with oral mucositis in children with malignancy [5].

Besides MTHFR also variants in TYMS have been frequently studied. We recently studied two common SNPs in the TYMS gene (rs2853542, 28 bp repeat 3RG >C and rs151264360, 6 bp del TTAAAG), which are involved in pyrimidine synthesis. A meta-analysis of four studies showed that the TYMS $2 \mathrm{R} 3 \mathrm{R}$ and $3 \mathrm{R} 3 \mathrm{R}$ genotypes were not significantly associated with acute lymphoblastic leukemia (OR: 1.45 [0.99-2.14]) [6]. In addition, SNPs in miRNA coding genes have been studied and the miR-1206 genetic variant (rs2114358) has been associated with oral mucositis in acute lymphoblastic leukemia patients [7,8]. Several other variants in PK/PD have been studied 
like genetic variation in SLC19A1, also known as reduced folate carrier (SLC19A1/RFC) and FPGS in relation to MTX plasma levels or oral mucositis, but most studies need replication to confirm their role in MTX-induced oral mucositis toxicity $[9,10]$.

In addition to genetic variants, we recently assessed changes in DNA methylation in relation to MTX-induced oral mucositis. DNA methylation is dependent upon the availability of methyl groups, which are donated through folatedependent one-carbon metabolism. MTX not only inhibits purine- and pyrimidine synthesis but also inhibits onecarbon metabolism $[11,12]$. As one-carbon metabolism donates methyl groups for all cellular methylation reactions, it can be hypothesized that MTX leads to impaired DNA methylation. Global DNA methylation was assessed by LINE-1 elements, which are a proxy for global DNA methylation, and in addition, two important metabolites of one-carbon metabolism, which are involved in the regulation of cellular methylation (S-adenosylmethionine [SAM] and s-adenosylhomocysteine [SAH]), were measured in acute lymphoblastic leukemia (ALL) patients before and after MTX therapy. Interestingly, plasma SAM levels increased and LINE-1 methylation decreased during MTX therapy but this was not associated with MTX-induced oral mucositis in ALL patients [3]. Future epigenome-wide association studies are of interest to assess the involvement of DNA methylation in MTX-induced oral mucositis.

\section{Challenges in pharmacogenetics of methotrexate-induced oral mucositis}

Pharmacogenetics has been shown useful in predicting toxicity of drugs influencing pyrimidine and purine synthesis, like 5-fluorouracil, by genetic variants in the gene coding for DPYD and azathioprine by genetic variants in TPMT $[13,14]$. However, MTX-induced toxicity is a complex interaction between disease and drug response, in which folate-rescue therapy is also given, which is relevant in predicting MTX toxicity. Most studies focused on pharmacogenetics of MTX-induced oral mucositis as an end point. We and others have developed methods to measure MTX and its polyglutamates (MTX-PG) intracellularly in erythrocytes using tandem mass spectrometry, which is of potential interest to study in relation to oral mucositis [15]. One recent genome-wide association study investigated MTX clearance from plasma and identified SLCO1B1 in children with ALL [16,17], which seems promising in MTX-induced oral mucositis [18]. However, genetic association studies of MTX toxicity are hampered by low sample size, which enables discovery of genetic variants, but often lacks replication of genetic loci. In future, functional studies are warranted that assess the role of genetic variants in relation to intermediate phenotypes such as (intracellular) MTX levels and clinical end points such as oral mucositis. In addition, next-generation sequencing of $\mathrm{PK} / \mathrm{PD}$ genes is a valuable approach to identify rare genetic variants that could predict MTX toxicity in a more personalized way.

\section{Financial \& competing interests disclosure}

The authors have no relevant affiliations or financial involvement with any organization or entity with a financial interest in or financial conflict with the subject matter or materials discussed in the manuscript. This includes employment, consultancies, honoraria, stock ownership or options, expert testimony, grants or patents received or pending, or royalties.

No writing assistance was utilized in the production of this manuscript.

\section{References}

1. Relling MV, Fairclough D, Ayers D et al. Patient characteristics associated with high-risk methotrexate concentrations and toxicity. J. Clin. Oncol. 12(8), 1667-1672 (1994).

2. Assaraf YG. The role of multidrug resistance efflux transporters in antifolate resistance and folate homeostasis. Drug Resist. Updat. 9(4-5), 227-246 (2006).

3. Oosterom N, Griffioen PH, den Hoed MAH et al. Global methylation in relation to methotrexate-induced oral mucositis in children with acute lymphoblastic leukemia. PLoS ONE 13(7), e0199574 (2018).

4. Frosst P, Blom HJ, Milos R et al. A candidate genetic risk factor for vascular disease: a common mutation in methylenetetrahydrofolate reductase. Nat. Genet. 10(1), 111-113 (1995).

5. Zhu C, Liu YW, Wang SZ et al. Associations between the C677T and A1298C polymorphisms of MTHFR and the toxicity of methotrexate in childhood malignancies: a meta-analysis. Pharmacogenomics J. 18(3), 450-459 (2018).

6. Oosterom N, Berrevoets M, den Hoed MAH et al. The role of genetic polymorphisms in the thymidylate synthase (TYMS) gene in methotrexate-induced oral mucositis in children with acute lymphoblastic leukemia. Pharmacogenet. Genomics 28(10), 223-229 (2018).

7. Gutierrez-Camino A, Oosterom N, den Hoed MAH et al. The miR-1206 microRNA variant is associated with methotrexate-induced oral mucositis in pediatric acute lymphoblastic leukemia. Pharmacogenet. Genomics 27(8), 303-306 (2017). 
8. Lopez-Lopez E, Gutiérrez-Camino Á, Piñán MÁ et al. Pharmacogenetics of microRNAs and microRNAs biogenesis machinery in pediatric acute lymphoblastic leukemia. PLoS ONE 9(3), e91261 (2014).

9. Suthandiram S, Gan GG, Zain SM et al. Effect of polymorphisms within methotrexate pathway genes on methotrexate toxicity and plasma levels in adults with hematological malignancies. Pharmacogenomics 15(11), 1479-1494 (2014).

10. den Hoed MA, Lopez-Lopez E, te Winkel ML et al. Genetic and metabolic determinants of methotrexate-induced mucositis in pediatric acute lymphoblastic leukemia. Pharmacogenomics J. 15(3), 248-254 (2015).

11. Broxson EH, Jr, Stork LC, Allen RH, Stabler SP, Kolhouse JF. Changes in plasma methionine and total homocysteine levels in patients receiving methotrexate infusions. Cancer Res. 49(21), 5879-5883 (1989).

12. Wang YC, Chiang EP. Low-dose methotrexate inhibits methionine S-adenosyltransferase in vitro and in vivo. Mol. Med. 18, 423-432 (2012).

13. Amstutz U, Henricks LM, Offer SM et al. Clinical Pharmacogenetics Implementation Consortium (CPIC) guideline for dihydropyrimidine dehydrogenase genotype and fluoropyrimidine dosing: 2017 update. Clin. Pharmacol. Ther. 103(2), 210-216 (2018).

14. Relling MV, Klein TE. CPIC: Clinical Pharmacogenetics Implementation Consortium of the Pharmacogenomics Research Network. Clin. Pharmacol. Ther. 89(3), 464-467 (2011).

15. den Boer E, Heil G, van Zelst BD et al. A U-HPLC-ESI-MS/MS-based stable isotope dilution method for the detection and quantitation of methotrexate in plasma. Ther. Drug Monit. 34(4), 432-439 (2012).

16. Trevino LR, Shimasaki N, Yang W et al. Germline genetic variation in an organic anion transporter polypeptide associated with methotrexate pharmacokinetics and clinical effects. J. Clin. Oncol. 27(35), 5972-5978 (2009).

17. Ramsey LB, Panetta JC, Smith C et al. Genome-wide study of methotrexate clearance replicates SLCO1B1. Blood 121(6), 898-904 (2013).

18. Liu SG, Gao C, Zhang RD et al. Polymorphisms in methotrexate transporters and their relationship to plasma methotrexate levels, toxicity of high-dose methotrexate, and outcome of pediatric acute lymphoblastic leukemia. Oncotarget 8(23), 37761-37772 (2017). 